\title{
Strategies of Starting and Stopping Antiepileptic Drugs in Patients With Seizure or Epilepsy; A Comprehensive Review
}

\author{
Majid Ghaffarpour ${ }^{1,{ }^{*}}$; Hossein Ali Ghelichnia ${ }^{1}$; Mohammad Hossein Harrichian ${ }^{1}$; Mojdeh \\ Ghabaee ${ }^{1}$; Mohammad Mahdi Saber Tehrani ${ }^{1}$; Parviz Bahrami ${ }^{2}$ \\ 1 Iranian Center of Neurological Research, Neuroscience Institute, Tehran University of Medical Sciences, Tehran, IR Iran \\ ${ }^{2}$ Department of Neurology, Lorestan University of Medical Sciences, Khorramabad, IR Iran \\ *Corresponding author: Majid Ghaffarpour, Neurology Department, Iranian Center of Neurology Research, Neuroscience Institute, Imam Khomeini Hospital, Tehran University of \\ Medical Sciences, Keshavarz Blv, Tehran, IR Iran. P.O. Box: 1419733141. Tel: +98-2166912274, Fax: +98-2166581558, E-mail: ghafarpour@sina.tums.ac.ir \\ Received: September 25, 2013; Revised: November 30, 2013; Accepted: December 29, 2013
}

\begin{abstract}
Context: Seizure is a transient neurologic dysfunction caused by sudden abnormal firing of cerebral neurons. Epileptic syndrome is a complex of symptoms and signs that is characterized by not only the etiology but also the age at onset, seizure type, pattern of electroencephalography and prognosis.

Evidence Acquisition: Treatment of the first seizures depends on their nature; some of them need no antiepileptic drugs (AED) therapy whereas the others should be treated appropriately. AEDs have long- and short-term adverse effects, interaction with other medications, and occupational and/or psychological consequences. Therefore, discontinuation of AEDs should be considered after a free-seizure period. Results: Studies show that the rate of seizure recurrence after AEDs withdrawal is about two to three times more than those who continue therapy; thus, the most difficult decision facing a neurologist is when to start medication and when/how it should be discontinued. Conclusions: This review explores the literature to answer above questions.
\end{abstract}

Keywords:Seizures; Epilepsy; Anticonvulsants

\section{Context}

Epilepsy or recurrent unprovoked seizures is a common chronic disorder with a prevalence of $0.5 \%$ to $0.8 \%$. It is slightly more common in males and in those with low socioeconomic status. The incidence of epilepsy is $0.03 \%$ to $0.05 \%$. Its cumulative incidence by the age of 75 years and the lifetime incidence are reported $2 \%$ to $3 \%$ and $4 \%$, respectively (1-5). In the United States, $0.65 \%$ of population is affected by active epilepsy whose age adjusted annual incidence is between $0.031 \%$ and $0.057 \%$. The cumulative lifetime incidence of seizures is $9 \%$ to $10 \%$, two third of which are symptomatic and the remainder are epileptic.

\section{Evidence Acquisition}

Management and treatment of patients with seizure comprise treatment of the first seizure and epilepsy as well as discontinuation of antiepileptic drugs (AEDs). While beneficial, AEDs have long- and short-term adverse effects, interaction with other medications, as well as occupational and/or psychological consequences; thus, their discontinuation should be considered after a freeseizure period, typically one to two years for children and two to five years for adults. Studies showed that the rate of seizure recurrence after AEDs withdrawal is about two to three times more than those who continue therapy (610). Therefore, the most important issue is when therapy should be started and when/ how it should be discontinued.

Seizure is a transient neurologic dysfunction (change in behavior, sensory conception, motor control, level of consciousness, cognition, and/ or autonomic function) caused by sudden abnormal firing of cerebral neurons. This aberrant electrical activity results from biochemical processes at the cellular level that promotes neuronal hyperexcitability as well as hypersynchrony and represents an imbalance between excitatory and inhibitory currents within the brain (11-14). The International League Against Epilepsy (ILAE) Task Force classified epilepsies based on both clinicoelecrographical features (1981) and syndromic patterns (1989). Epileptic syndrome is a complex of signs and symptoms defining a unique epilepsy condition that is characterized not only by the etiology but also by the age at onset, seizure type, electroencephalography (EEG) patterns, and prognosis.

\section{Results}

Appropriate treatment of patients with seizure or epi- 
lepsy needs considering situations upon which seizure or epilepsy happens.

\subsection{Treatment of the First Seizure}

Treatment of the first seizures depends on their nature. Some of them need no AED therapy, whereas others should be treated appropriately.

\subsubsection{Acute Non-Structural Symptomatic Seizures}

These seizures occur in close temporal association with acute neurologic and medical conditions such as withdrawal from hypnotic/sedatives or alcohol, dehydration, fever, hypocalcaemia, hypoglycemia or hyperglycemia, hyponatremia or hypernatremia, uremia, hypomagnesaemia, use of drugs, particularly cocaine and other CNS stimulants (Table 1), or presenting as post impact (immediate) seizure following head injury.

In these circumstances, management is directed toward identification and treatment of the underlying illness and if AEDs are needed to suppress seizures acutely, they generally are not needed to be continued after the illness subsides.

\begin{tabular}{|c|}
\hline Antimicrobial/Antivirals \\
\hline Acyclovir/Ganciclovir \\
\hline$\beta$-lactam and related compounds \\
\hline Isoniazid \\
\hline Quinolones \\
\hline Analgesics/Anesthetics \\
\hline Local anesthesia \\
\hline Meperidine \\
\hline Tramadol \\
\hline Drugs of abuse \\
\hline Amphetamine \\
\hline Cocaine \\
\hline Methylphenidate \\
\hline Phencyclidine (PCP or angel dust) \\
\hline Psychotropics \\
\hline Antidepressants \\
\hline Antipsychotics \\
\hline Lithium \\
\hline Radiographic contrasts \\
\hline Sedative/ hypnotics \\
\hline $\begin{array}{l}\text { Alcohol, barbiturates or flumazenil in benzodiazepines- } \\
\text { dependent patients (benzodiazepines withdrawal ) }\end{array}$ \\
\hline
\end{tabular}

\subsubsection{Febrile Convulsion}

Febrile Convulsion (FC) is defined by ILAE (1993) as a seizure in association with febrile illness in the absence of
CNS infection or acute electrolyte imbalance in children older than one month without prior afebrile seizure. It is the most common cause of convulsion in children that affects $2 \%$ and $4 \%$ of children in USA and Europe, respectively, and $10 \%$ of children in Japan. Two third of FCs occur in the first 24 hours of illness. FC occurs mostly between three months and six years of age and $80 \%$ of cases are a benign, single, or isolated brief (1-2 min) tonic-clonic seizure (simple FC). Complex FCs is defined by criteria of being focal, Todd's paralysis, seizure with duration of 10$15 \mathrm{~min}$, and presence of more than one episode within 24 hours. The risk of recurrent epilepsy in children with FC is similar to that of general population but increases if FC is repeated. Children with complex FC have a greatly increased risk of recurrence being $8 \%, 17 \%$, and $49 \%$ depending on the association of one, two, or three complicating features respectively $(15,16)$. The complex FC, preexisting neurodevelopmental abnormalities, positive family history of epilepsy, and recurrent febrile seizures predict later epilepsy $(15,16)$.

Even when recurrent, FCs represent acute symptomatic seizures and are diagnosed as epilepsy. Simple FCs have no long-term adverse results and prophylactic AED should be avoided even after two or three isolated convulsions. Although Valproate (VLP) and Phenobarbital (PHB) reduce recurrence, they have no effect on the risk of later epilepsy. Phenytoin (PHT) and Carbamazepine (CBZ) are ineffective. Finally, if AED is considered, it should be limited to children with high risk of developing epilepsy (as previously mentioned) or a history of prolonged febrile seizures.

More than $90 \%$ of alcohol withdrawal seizures (Rum Fits) occur as grand mal seizures during the 7-48 hours following the cessation of drinking or within 48 hours of the last alcohol intake with a peak incidence between 13-24 hours (17-19). They may present as a single attack, but usually occur in bursts of two to six over a day and rarely (2\%) as status epilepticus (SE). These seizures, either single or brief flurry in type, are usually self-limited and need no antiepileptic therapy except recurrent or prolonged ones, in which short-term AED is recommended. In the latter situations, benzodiazepines (BZDs) or PHB are preferred. PHT has no effect (20). The rare instances of SE should be treated like status from other etiologies.

\subsubsection{Posttraumatic Seizures}

Posttraumatic seizures (PTS) occur in approximately 5\% of closed head injuries as well as 50\% of patients with a compound skull fracture and direct wounds of the brain. Depending on the interval between head injury and the seizures, post injury seizures are divided into three types. Immediate (post impact) seizures present within moments of the injury, usually as a brief tonic extension of extremities with shaking movements, followed by awakening in a mild confusional state. These seizures have good prognosis and some authors tend not to treat them (18). 


\subsubsection{Uremic Encephalopathy Seizure}

Convulsions occur in one third of patients with uremia, often in preterminal patients; they should be treated when observed. Patients with fluctuating level of consciousness should have an EEG to rule out nonconvulsive Status epilepticus (SE). Uremic encephalopathy associated with acute renal failure may cause seizures due to hyponatremia, calcium disorders, uremia, or disequilibrium syndrome seen with hemodialysis. The incidence of seizures in the latter condition is reported to be $2 \%$ to $10 \%$ (21). These seizures may be resistant to treatment until the uremia is appropriately treated. However, some seizures may respond to relatively low plasma concentrations of AEDs because the serum albumin is depressed in uremia, increasing the unbound, therapeutically active portion of a drug; therefore, blood levels of unbound AED should be measured. If there is hyponatremia, the seizure may be difficult to control.

Some carbonic anhydrase inhibitors (zonisamide and topiramate) are relatively contraindicated in patients at risk of nephrolithiasis or when etiology of the renal failure is not clear. Gabapentin, pregabalin, levetiracetam, $\mathrm{PHB}$, and topiramate are eliminated by the kidneys; thus, they should be used in lower doses in patients with renal diseases. Some of AEDs (Ethosuximide, pregabalin, gabapentin, levetiracetam, and to some extent, PHB and topiramate) are removed by dialysis and their serum levels decrease by $50 \%$, therefore, replacement after dialysis is required.

\subsection{Structural Lesion-Induced Symptomatic Sei- zures}

Several pathologies including brain infections (abscesses and herpes encephalitis), trauma, arteriovenous malformations (AVMs), primary brain tumors as well as metastases, and stroke may induce recurrent seizures and sometimes SE that need immediate treatment.

Brain tumors are common causes of epilepsy, particularly drug resistant seizures. Most of these epilepsies are related to low grade tumors, especially those in temporal lobe. Type of tumor and its location are important factors. High-grade gliomas, meningiomas, and brain metastases (except melanoma metastases) less frequently induce seizure in contrast to gangliomas, dysembryoplastic neuroepithelial tumors (DNETs), and low-grade glioma. Cortical tumors are more likely to cause seizure than those in other locations.

AED is advised in all cases with brain tumor who had a seizure; however, prophylactic therapy does not protect these patients against further seizures (19). Thus, except in unusual circumstances such as melanoma metastases, tumors of frontal cortex, or concomitant metastases, which have a higher risk of seizures, AED is not recommended. This idea is based on not only lack of prophylactic effect but also the fact that the patients with brain tumors are at high risk of AEDs adverse effects (19-22). It is preferable to use nonhepatic enzyme-inducer drugs. PHB, PHT, and CBZ are enzyme inducers and thus, enhance metabolisms of many chemotherapeutic agents and decrease their efficacies. In contrast, VLP results in toxicity during chemotherapy.

Enzyme-inducer drugs may cause rash and StevenJohnson syndrome. The risk of the latter syndrome in cases receiving PHT is enhanced by external brain radiotherapy. For this reason, PHT should be changed before radiation. Levetiracetam is a non-CYP enzyme-inducer drug (23) that is approved to be effective in brain tumorrelated epilepsy. PHT inhibits insulin release and may cause hyperosmolar nonketotic hyperglycemia (as does steroids, thiazide, or infection) in patients with diabetes. This syndrome arises usually in conjunction with the combined use of the PHT and steroid, for example, in elderly patients with brain tumor. Postoperative seizure prophylaxis is controversial; however, it is typically initiated intraoperatively and is maintained up to one week following surgery. Although such therapy has not been proved to decrease the incidence of postoperative seizures, it prevents seizures that might increase morbidity and mortality $(21,24)$.

Severe traumatic brain injury accounts for $20 \%$ of symptomatic epilepsies that need to be treated, although they are poorly responsive. In contrast to immediate PTS, early seizures that occur within seven days after trauma have a strong risk of developing later epilepsy; however, it is believed that this is usually related to the severity of the head trauma. Studies have shown that prophylactic therapy had no proven effect (25); however, PHT was effective in preventing seizure in the first week (26). New AEDs have not been tested for the prevention of PTS. In general, AEDs are used to prevent early seizures but should not be given more than two weeks because they do not decrease the frequency of late PTS.

Among vascular malformation, epilepsy occurs most commonly in association with AVMs and cavernous malformations. Venous malformation is not directly related to epilepsy unless associated with a cavernous type. Small AVMs are more likely to present with epilepsy, whereas larger AVMs usually result in bleeding.

On magnetic resonance imaging, cavernous malformations have characteristic popcorn appearance with mixed signal and a rim of decreased signal (hemosiderin). They are strongly associated with epilepsy and have small risk for bleeding. In contrast to AVMs, if cavernous malformation-induced seizures are controlled with AED, there is no clear indication for surgery except when the epilepsy is drug-resistant. Resection yields good results, provided that hemosiderin-stained brain tissue is also removed (27).

Studies show that the frequency of seizures in stroke ranges between $2.3 \%$ and $14 \%$. Stroke-induced early seizures are defined as those that occur within 14 days of the stroke and late seizures are those that occur after this period 
(28). They may occur at any age and are the most common cause of seizures in the elderly. As in PTS, early seizures most often do not progress to chronic epilepsy, but due to increased risk of late epilepsy (approximately, 1/3 of cases with early poststroke seizures develop late epilepsy), antiepileptic therapy is recommended. Prophylactic anticonvulsants for seizures after a typical embolic or thrombotic type stroke and cerebral hemorrhage is not necessary (18).

Seizures are a major complication of intracranial hemorrhage (ICH), usually developing within 24 hours of hemorrhage. It is reasonable to begin PHT or VLP immediately. If no seizure occurred, prophylactic therapy is usually discontinued, although there are some different ideas about its time (from one to six months after the ICH). Certainly, those with later epilepsy should receive long-term therapy (28).

Obvious tonic-clonic seizures occur in $50 \%$ of patients with subarachnoid hemorrhage during hospitalization and in another $10 \%$ in the first year after discharge. Ictal events at the onset of bleeding do not foreshadow an increased risk of late seizures (29). Others noted that seizures occur in up to $20 \%$ of patients with SAH during the first two weeks, whereas only $6 \%$ to $8 \%$ develop late epilepsy. Prophylaxis with intravenous PHT $(20 \mathrm{mg} / \mathrm{kg}$ ) to achieve the therapeutic levels (15-20 ng/dL) should be maintained throughout their admission. VLP is also effective. In uncomplicated cases, AED can be stopped after the first month, but may be used longer if there is a high likelihood of developing epilepsy such as cerebral infarction or brain abscess (28). Short-term prophylaxis is recommended in the case of convulsive seizure; however, there is controversy in the case of nonconvulsive seizure in these patients (30).

\subsection{Remote Symptomatic Seizures}

Remote Symptomatic Seizures (RSSs) occur in patients with a history of stroke, trauma, CNS infection, or static encephalopathy from birth with mental retardation or cerebral palsy. Multiple seizures or SE at onset, Todd's postictal paresis, and prior acute seizure increase the risk of recurrence seizure in this group. Antiepileptic therapy is needed if above mentioned risk factors exist.

\subsection{Single Unprovoked Seizure}

A two-year recurrence rate of first unprovoked seizure is reported between $39 \%$ (31) and 51\%(9). Treatment reduced the risk by $60 \%$ in one study (31) and by $30 \%$ in another (8). Abnormal EEG and neurological status most influenced recurrence rate (32). In spite of these facts, there is no evidence that therapy alters the prognosis of epilepsy. Therefore, treatment should not be advised without having details in mind and decision to treat should be made only in consultation with the patient or parents after weighing the unique circumstances posed by the individual. However, in most cases with idiopathic first seizure, deferring treatment until a second seizure is a reasonable and often preferable decision (29).
Most neurologists treat first unprovoked seizures in patients with high risk of recurrence such as history of epilepsy in siblings (not parents), history of postictal Todd's paresis, multiple seizures at onset, and history of brain injury, stroke, CNS infection, or significant head trauma.

Single seizure treatment was categorized by International League Against Epilepsy (ILAE) Leppike (33) as the following:

I - Definitely needs treatment

a - With structural lesion such as brain tumors, brain abscess, herpes encephalitis, and

AVMs or cavernous malformation

b - Without structural lesion, but with high risk of recurrence:

- History of epilepsy in siblings (not parents)

- History of Todd's postictal paresis

- Multiple seizures or SE at onset

- Prior acute seizure

- History of brain injury (stroke, infection, significance)

- EEG with definite epileptic pattern, especially generalized spike-wave complexes

II - Possibly needs treatment

- Unprovoked seizure with none of the above risk factors, but with special conditions such as need to drive or work at height

III - Probably needs no treatment (also short-term therapy may be used)

- Alcohol withdrawal

- Acute symptomatic seizures

- Post impact seizures

- Seizure provoked by excessive sleep deprivation

- Specific benign epileptic syndromes such as simple febrile convulsion, benign epilepsy with centrotemporal spikes (BECTS), or Childhood Absence Epilepsy (CAE)

After few nocturnal seizures, BECTS remit spontaneously by adolescence in $90 \%$ of cases. On the other hand, CAE may go into remission in $55 \%$ of children, especially in those without secondary generalized tonic clonic seizure; thus, may not need treatment.

\subsection{Two Unprovoked Seizures}

After two unprovoked seizures, the risk of recurrence increases markedly (61\% at 2 years and $73 \%$ at 5 years). Furthermore, diagnosis of epilepsy is made following second unprovoked seizure; therefore, antiepileptic treatment is necessary.

\subsubsection{Discontinuation of Antiepileptic Drugs}

According to the report of Kwan and Brodie (34), approximately two third of epileptic patients achieve seizure freedom with appropriately chosen treatment. Unlike other medications that usually have a well-defined course, duration of antiepileptic therapy remained somewhat controversial. Due to adverse effect of AEDs, strategy for discontinuation of AEDs (D-AEDs) should be considered after a period of seizure freedom (typically two to five years in adults and one to two years in children) 
except in patients who have epilepsy due to progressive neurologic disorder. Before decision to withdraw AED, patient should be informed about risk for relapse and the potential implications of recurrence seizures, particularly for safety and driving. Some patients fear the consequences of withdrawing and are apt to continue medication while others are willing to stop it even when the risk of recurrence is high. The rate of seizure recurrence following D-AEDs is two to three times the rate in those who continue treatment $(7,8,32,35)$. The average recurrence rates in children and adults were reported $31.2 \%$ and $39.4 \%$, respectively (36). Recurrent seizures occur relatively soon after D-AEDs, half of which take place within six months and the entire at the first two years.

\subsubsection{Prognostic Factors Influencing Recurrence Rate}

All studies in adults including Medical Research Council (MRC) Antiepileptic Drug Withdrawal Study Group (37) confirmed an increased risk of seizure recurrence following D-AEDs, even after a seizure-free period. Some studies $(38,39)$ found that an abnormal neurologic examination, IQ $<70$, longer duration of epilepsy, higher number of seizures, and multiple type seizures are associated with increased risk of recurrence. The MRC study (37) reported that a higher risk of recurrence was correlated with both the prior use of multiple AEDs and generalized tonic clonic seizure type. Moreover, focal epileptiform discharges on EEG and worsening of the EEG after D-AEDs are other unfavorable factors (40-42). Finally, focal findings on magnetic resonance imaging such as hippocampal pathology increase the risk of recurrence (43). In children population epilepsy, there are additional special factors that should be considered during decision to withdraw AEDs:

- Recurrent rate is slightly lower in children than in adults.

- Natural course of some epileptic syndromes is benign; they remit regardless of treatment and whereas, $10 \%$ of them including juvenile myoclonic epilepsy (JME) and reading epilepsy will never remit.

- In a review at 2008, a seizure-free period of one to two years was recommended as an acceptable period for justified AED withdrawal (44).

- Adolescent age of onset $(45,46)$, a symptomatic etiology, an abnormal neurological exam, and epileptiform discharge before or after withdrawal increase the risk of recurrence.

In general, favorable and unfavorable factors for D-AEDs are listed in Table 2.

Table 2. Favorable and Unfavorable Risk Factors for Discontinuation of Antiepileptic Drugs

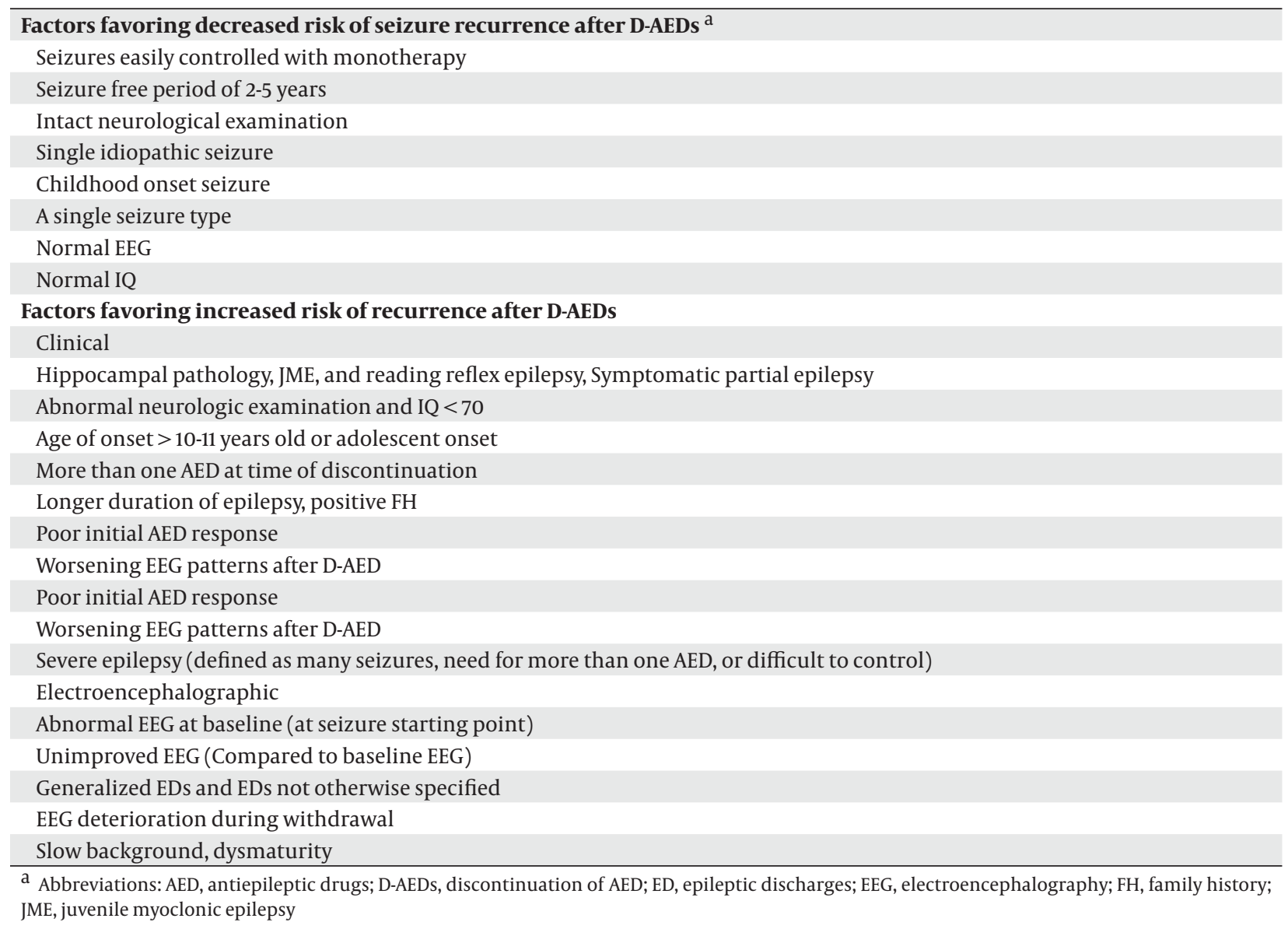




\subsection{Pace of Antiepileptic Drugs Tapering}

Few studies focused on the methods of withdrawing AEDs and there is a little evidence in favor of particular style of medication tapering. Some authors defined rapid taper as occurring within three months or less and slow taper as occurring longer than three months (47). In the Cochrance review, the tapering period in children ranged from one month to one year. In a study by Serra et al. no difference was found between tapering periods of one month and six months (48).

Although meeting the methodological criteria and defining the optimal tapering period is even much insufficient in adult studies, occurrence of seizures relapse mostly within the six months after D-AEDs, safety, and quality of life issues are all in favor of relatively rapid (1-3 months) tapering.

When discontinuation of treatment is elected, slow drug tapering is mandatory because rapid withdrawal may result in withdrawal seizures or even SE. This principle is particularly true for PHB and BZDs, particularly Clonazepam, that have long half-life and great potential for causing withdrawal seizures. These drugs should be withdrawn over many months, usually in increment of $25 \%$ of the initial doses. PHT, CBZ, and VLP can be withdrawn over six to ten months. More prolonged withdrawal probably does not reduce the risk of seizure recurrence.

\subsection{Benefits and Risks of Treatment Discontinua- tion}

D-AEDs eliminate short- and long-term adverse effects of AEDs. In addition some studies have documented that mood and psychiatric condition of patients may improve after drug discontinuation $(49,50)$. Conversely, in patients who have comorbid bipolar disorder or headache, VLP and lamotrigine withdrawal may have negative effect on psychiatric state of patients and withdrawal from topiramate may worsen the headache. For many patients, these negative effects are enough to prevent them from drug discontinuation. A few studies focused on the possibility of the development of resistant seizures or sudden unexplained death following drug discontinuation during recurrence phase, but this is not usually the case For example, Comfield and Comfield found that 97\% of relapsed seizures in children were controlled again . This is less clear in adults (42).

\subsection{Role of Electroencephalography in Decision to Start or to Stop Antiepileptic Drug Treatment}

Although EEG plays an important role in the diagnosis of seizure disorders and may predict outcome of the treatment discontinuation, role of EEG as a predictor of seizure recurrence after D-AED is still an issue of debate because of its weak points and limitations. Weak points include:
1) EEG findings, like those of EKG and EMG, are meaningful only in relation to the illness under consideration and the clinical state of patient during recording.

2) Some of epileptic patients have normal interictal EEG, even during the aura phase of a simple or complex partial seizure (CPS). Conversely, a few normal individuals may have abnormal EEG. In addition, similar EEG abnormalities may build up in conditions other than epilepsy. For example, epileptiform discharges and rarely periodic lateralized epileptiform discharges (PLEDs) can be found in patients with migraine (51).

3) Differentiation between normal patterns that may simulate seizure and the true epileptic or interictal discharges is still a challenge for those who report EEG.

4) Although gross EEG abnormalities are by themselves undoubtedly abnormal, lesser degrees of abnormalities, form a continuum between abnormal and the completely normal EEG, are less significant (52).

5) In a study of intracranial and scalp recordings, only $10 \%$ of discharges involving less than $10 \mathrm{~cm}^{2}$ of cortical surface area were detectable as an interictal spike on scalp EEG, while none were detectable in those involving less than $6 \mathrm{~cm}^{2}$ of the cortex (53).

6) Underlying cause of seizure, type of the epilepsy syndrome, and pharmacologic effects of AED therapy are other factors influencing the value of the EEG in the setting of D-AED.

Despite above limitations, EEG may be helpful by:

1) Detecting abnormal brain function not apparent on the neurological examination.

2) Predicting the possibility of seizure relapse after DAED.

3) Facilitating diagnosis of epileptic syndromes and therefore, giving appropriate advice about D-AED. Rolandic or Centro temporal spikes in patients with rolandic epilepsy and $3 \mathrm{~Hz}$ spike-wave discharges in children with CAE are benign findings. Conversely, anterior temporal sharp waves (indicating temporal lobe epilepsy), generalized slow spike-wave pattern, frontal spikes in patient with partial frontal epilepsy, and generalized polyspikewave discharges in JME have higher rate of intractability and lower remission rates. Many patients have not specific epileptic syndrome or specific epileptogenic discharges and hence, will not benefit from EEG.

\subsection{Gross Electroencephalographic Abnormalities}

Focal diminished or absent brain waves:

This pattern is seen with infarction, tumors, traumatic necrosis, or extensive clot between cerebral cortex and electrode. However, most of these lesions are not large enough to abolish brain waves and the EEG may record abnormal high voltage (50-350 mV) slow waves arising from functioning brain at the border of the lesion.

Focal fast activity:

It is recorded in patients with skull defect or early phase of brain ischemia as well as at the beginning of 
some seizures.

Epileptiform discharges (spikes, sharps, and spike-wave complexes):

These are presented interictally in patients with epilepsy or in individuals with a genetic predisposition to seizure.

Burst-suppression pattern and flat EEG (electrocerebral silence).

\subsection{Normal Variants or Borderline EEG}

Borderline findings have no clinical significance in an otherwise normal person, but become meaningful in those with particular signs and symptoms. They include:

- Fourteen-Hertz and 6-Hz positive spikes or small sharp waves during sleep.

- Scattered 5-Hz or 6-Hz slowing.

- Minor voltage asymmetries.

- Persistence of breakdown (appearance of 3-4 Hz activity during middle and later part of hyperventilation in children) for a few minutes after hyperventilation.

\section{Discussion}

Keeping in view the considerations that: 1$)$ some of the seizures and epileptic syndromes are benign and selflimited , 2) the majority of the AEDs have serious adverse effects, resulting sometimes in undesirable consequences and 3) seizures may relapse following treatment withdrawal, physician should know much more about when to start and how or when to stop anti-epileptic therapy.

\section{Acknowledgements}

We thank Mrs. Maryam Rashidi in co-operation of this article.

\section{Authors' Contribution}

None declared.

\section{Financial Disclosure}

There is no financial support

\section{Funding/Support}

There is no fund.

\section{References}

1. Jallon P. Epilepsy and epileptic disorders, an epidemiological marker? Contribution of descriptive epidemiology. Epileptic Disord. 2002;4(1):1-13.

2. Hauser WA. Epidemiology of epilepsy in children. Neurosurg Clin NAm.1995;6(3):419-29.

3. Samuels MA, Ropper AH. Samuel's Manual of Neurologic Therapeutics. 9th edPhiladelphia: Lippincott Williams \& Wilkins; 2010.

4. Warlow C, Hankey GJ. The Lancet Handbook of Treatment in Neurology.Edinburgh: Elsevier; 2006.

5. Hauser WA, Hesdorffer DC, Epilepsy Foundation of America . Epilepsy: frequency, causes and consequences.: Demos; 1990.

6. Hauser WA, Rich SS, Lee JR, Annegers JF, Anderson VE. Risk of recurrent seizures after two unprovoked seizures. $N$ Engl J Med. 1998;338(7):429-34.

7. O'Dell C, Shinnar S. Initiation and discontinuation of antiepileptic drugs. Neurol Clin. 2001;19(2):289-311.

8. Marson A, Jacoby A, Johnson A, Kim L, Gamble C, Chadwick D, et al. Immediate versus deferred antiepileptic drug treatment for early epilepsy and single seizures: a randomised controlled trial. Lancet. 2005;365(9476):2007-13.

9. Berg AT. Risk of recurrence after a first unprovoked seizure. Epilepsia. 2008;49 Suppl 1:13-8.

10. Schmidt D. AED discontinuation may be dangerous for seizurefree patients. J Neural Transm. 2011;118(2):183-6.

11. Fisher RS, van Emde Boas W, Blume W, Elger C, Genton P, Lee P, et al. Epileptic seizures and epilepsy: definitions proposed by the International League Against Epilepsy (ILAE) and the International Bureau for Epilepsy (IBE). Epilepsia. 2005;46(4):470-2.

12. McCormick DA, Contreras D. On the cellular and network bases of epileptic seizures. Annu Rev Physiol. 2001;63:815-46.

13. Faingold CL. Emergent properties of CNS neuronal networks as targets for pharmacology: application to anticonvulsant drug action. Prog Neurobiol. 2004;72(1):55-85.

14. Chang BS, Lowenstein DH. Epilepsy. $N$ Engl $J$ Med. 2003;349(13):1257-66.

15. Annegers JF, Hauser WA, Shirts SB, Kurland LT. Factors prognostic of unprovoked seizures after febrile convulsions. $N$ Engl J Med. 1987;316(9):493-8.

16. Tarkka R, Rantala H, Uhari M, Pokka T. Risk of recurrence and outcome after the first febrile seizure. Pediatr Neurol. 1998;18(3):21820.

17. Victor M. 33. The pathophysiology of alcoholic epilepsy. Res Publ Assoc Res Nerv Ment Dis. 1968;46:431-54.

18. Ropper A, Samuels M. Adams and Victor's Principles of Neurology. 9th edNew York: Mcgraw-hill; 2009.

19. Daroff RB, Bradley WG, Fenichel GM, Jankovic J, Mazziotta JC. Bradley's Neurology in Clinical Practice. 6th edPhiladelphia: Elsevier - Health Sciences Division; 2012.

20. Scorza FA, Albuquerque M, Arida RM, Cysneiros RM, Henriques TM, Scorza CA, et al. Seizure occurrence in patients with chronic renal insufficiency in regular hemodialysis program. Arq Neuropsiquiatr. 2005;63(3B):757-60.

21. Glantz MJ, Cole BF, Forsyth PA, Recht LD, Wen PY, Chamberlain MC, et al. Practice parameter: anticonvulsant prophylaxis in patients with newly diagnosed brain tumors. Report of the Quality Standards Subcommittee of the American Academy of Neurology. Neurology. 2000;54(10):1886-93.

22. Brunton LL, Gilman A, Goodman LS. Goodman and Gilman's The Pharmacological Basis of Therapeutics. 11th ed: McGraw-Hill; 2006.

23. Horwitz NH. Library: Historical Perspective. Neurosurgery. 1994;35(1):168.

24. Temkin NR. Preventing and treating posttraumatic seizures: the human experience. Epilepsia. 2009;50 Suppl 2:10-3.

25. Temkin NR. Neurology and Trauma.: Saunders; 1996.

26. Awad I, Jabbour P. Cerebral cavernous malformations and epilepsy. Neurosurg Focus. 2006;21(1).

27. Menon B, Shorvon SD. Ischaemic stroke in adults and epilepsy. Epilepsy Res. 2009;87(1):1-11.

28. Johnson RT, Griffin JW, McArthur JC. Current Therapy in Neurologic Disease. 7th ed: Elsevier Mosby; 2006.

29. Rowland LP, Pedley TA, Merritt HH. Merritt's Neurology. 12th ed: Lippincott Williams \& Wilkins; 2010.

30. Gilmore E, Choi HA, Hirsch LJ, Claassen J. Seizures and CNS hemorrhage: spontaneous intracerebral and aneurysmal subarachnoid hemorrhage. Neurologist. 2010;16(3):165-75.

31. Berg AT, Shinnar S. The risk of seizure recurrence following a first unprovoked seizure: a quantitative review. Neurology. 1991;41(7):965-72

32. Kim LG, Johnson TL, Marson AG, Chadwick DW, Mrc Mess Study group . Prediction of risk of seizure recurrence after a single seizure and early epilepsy: further results from the MESS trial. Lancet Neurol. 2006;5(4):317-22.

33. Leppik IE. Contemporary Diagnosis and Management of The Patient With Epilepsy.Newtown, Pensylvania, USA: Handbooks in Health 
Care; 2001.

34. Kwan P, Brodie MJ. Early identification of refractory epilepsy. $N$ Engl J Med. 2000;342(5):314-9.

35. Randomized clinical trial on the efficacy of antiepileptic drugs in reducing the risk of relapse after a first unprovoked tonicclonic seizure. First Seizure Trial Group (FIR.S.T. Group). Neurology. 1993;43(3 Pt 1):478-83.

36. Practice parameter: a guideline for discontinuing antiepileptic drugs in seizure-free patients--summary statement. Report of the Quality Standards Subcommittee of the American Academy of Neurology. Neurology. 1996;47(2):600-2.

37. Randomised study of antiepileptic drug withdrawal in patients in remission. Medical Research Council Antiepileptic Drug Withdrawal Study Group. Lancet. 1991;337(8751):1175-80.

38. Shih JJ, Ochoa JG. A systematic review of antiepileptic drug initiation and withdrawal. Neurologist. 2009;15(3):122-31.

39. Specchio LM, Beghi E. Should antiepileptic drugs be withdrawn in seizure-free patients? CNS Drugs. 2004;18(4):201-12.

40. Specchio LM, Tramacere L, La Neve A, Beghi E. Discontinuing antiepileptic drugs in patients who are seizure free on monotherapy. J Neurol Neurosurg Psychiatry. 2002;72(1):22-5.

41. Callaghan N, Garrett A, Goggin T. Withdrawal of anticonvulsant drugs in patients free of seizures for two years. A prospective study. N Engl J Med.1988;318(15):942-6.

42. Lossius MI, Hessen E, Mowinckel P, Stavem K, Erikssen J, Gulbrandsen P, et al. Consequences of antiepileptic drug withdrawal: a randomized, double-blind study (Akershus Study). Epilepsia. 2008;49(3):455-63.

43. Cardoso TA, Coan AC, Kobayashi E, Guerreiro CA, Li LM, Cendes F. Hippocampal abnormalities and seizure recurrence after antiepileptic drug withdrawal. Neurology. 2006;67(1):134-6.
44. Camfield P, Camfield C. When is it safe to discontinue AED treatment? Epilepsia. 2008;49 Suppl 9:25-8.

45. Berg AT, Shinnar S. Relapse following discontinuation of antiepileptic drugs: a meta-analysis. Neurology. 1994;44(4):601-8.

46. Verrotti A, Morresi S, Basciani F, Cutarella R, Morgese G, Chiarelli F. Discontinuation of anticonvulsant therapy in children with partial epilepsy. Neurology. 2000;55(9):1393-5.

47. Hixson JD. Stopping antiepileptic drugs: when and why? Curr Treat Options Neurol. 2010;12(5):434-42.

48. Serra JG, Montenegro MA, Guerreiro MM. Antiepileptic drug withdrawal in childhood: does the duration of tapering off matter for seizure recurrence? J Child Neurol. 2005;20(7):624-6.

49. Hessen E, Lossius MI, Reinvang I, Gjerstad L. Influence of major antiepileptic drugs on neuropsychological function: results from a randomized, double-blind, placebo-controlled withdrawal study of seizure-free epilepsy patients on monotherapy. J Int Neuropsychol Soc. 2007;13(3):393-400.

50. Hessen E, Lossius MI, Reinvang I, Gjerstad L. Slight improvement in mood and irritability after antiepileptic drug withdrawal: a controlled study in patients on monotherapy. Epilepsy Behav. 2007;10(3):449-55.

51. Pourmahmoodian H, Kahani M, Ghaffarpour M, Harrirchian MH, Ghabaee M, Fallah A. Ictal and interictal EEG abnormalities in 100 migraineurs with and without aura. Acta Medica Iranica. 2007;45(4):309-15.

52. Jallon P, Loiseau P, Loiseau J. Newly diagnosed unprovoked epileptic seizures: presentation at diagnosis in CAROLE study. Coordination Active du Reseau Observatoire Longitudinal de l' Epilepsie. Epilepsia. 2001;42(4):464-75.

53. Tao JX, Ray A, Hawes-Ebersole S, Ebersole JS. Intracranial EEG substrates of scalp EEG interictal spikes. Epilepsia. 2005;46(5):669-76. 